\title{
Analysis of skin blood microflow oscillations in patients with rheumatic diseases
}

\author{
Irina Mizeva \\ Irina Makovik \\ Andrey Dunaev \\ Alexander Krupatkin \\ Igor Meglinski
}




\section{Analysis of skin blood microflow oscillations in patients with rheumatic diseases}

\author{
Irina Mizeva, ${ }^{a, \star}$ Irina Makovik, ${ }^{\text {b }}$ Andrey Dunaev, \\ Alexander Krupatkin, ${ }^{c}$ and Igor Meglinski ${ }^{\mathrm{d}, \mathrm{e}, \mathrm{f,g}}$ \\ ${ }^{a}$ Institute of Continuous Media Mechanics, Perm, Russia \\ borel State University named after I.S. Turgenev, Orel, Russia \\ 'Priorov Central Scientific Research Institute of Traumatology and \\ Orthopedics, Moscow, Russia \\ dUniversity of Oulu, Opto-Electronics and Measurement Techniques, \\ Oulu, Finland \\ enstitute of Biology, Irkutsk State University, Irkutsk, Russia \\ fITMO University, St. Petersburg, Russia \\ ${ }^{9}$ Tomsk State University, Interdisciplinary Laboratory of Biophotonics, \\ Tomsk, Russia
}

\begin{abstract}
Laser Doppler flowmetry (LDF) has been applied for the assessment of variation in blood microflows in patients with rheumatic diseases and healthy volunteers. Oscillations of peripheral blood microcirculation observed by LDF have been analyzed utilizing a wavelet transform. A higher amplitude of blood microflow oscillations has been observed in a high frequency band (over $0.1 \mathrm{~Hz}$ ) in patients with rheumatic diseases. Oscillations in the high frequency band decreased in healthy volunteers in response to the cold pressor test, whereas lower frequency pulsations prevailed in patients with rheumatic diseases. A higher perfusion rate at normal conditions was observed in patients, and a weaker response to cold stimulation was observed in healthy volunteers. Analysis of blood microflow oscillations has a high potential for evaluation of mechanisms of blood flow regulation and diagnosis of vascular abnormalities associated with rheumatic diseases. () 2017 Society of Photo-Optical Instrumentation Engineers (SPIE) [DOI: 10.1117/1.JBO.22.7.070501]
\end{abstract}

Keywords: laser Doppler flowmetry; peripheral blood circulation; blood microflows; wavelet transfer; oscillations; rheumatic.

Paper 170324LR received May 25, 2017; accepted for publication Jun. 21, 2017; published online Jul. 13, 2017.

Blood microcirculation plays an important role in the transport of nutriments, hormones, and oxygen and the discharge of metabolic waste. The significance of systemic vasculature, as well as associated thermoregulation and autoregulatory mechanisms, attracts a continuously growing interest in the understanding of blood microcirculation.

The morphological microvascular structure changes with pathological deviations and abnormalities in vascular tone regulation appearing. ${ }^{1}$ Rheumatic diseases lead to microvascular morphological abnormalities and their reactive progression, including rarefication of the capillary network, capillaries asymmetry, and appearance of megacapillaries (with the diameter $\sim 50 \mu \mathrm{m}){ }^{2}$ The functional disorder of microcirculation in patients with rheumatic diseases (PRD) has been observed during the temperature test. ${ }^{3}$

Spontaneous oscillations in the tone of skin blood vessel walls independent of heart beat (known as vasomotion) are used for functional studies of blood microcirculation. It has been shown that skin vasomotion depends on various physiological mechanisms that cause pulsations at different frequency bands. ${ }^{4}$ Oscillations of skin blood flow observed in the frequency intervals 0.6 to $1.6 \mathrm{~Hz}$ and 0.2 to $0.6 \mathrm{~Hz}$ are associated with heart and respiratory activities, respectively, ${ }^{5-7}$ whereas oscillations in the $0.009-$ to $0.1-\mathrm{Hz}$ frequency band are related to myogenic, ${ }^{8-10}$ neurogenic, ${ }^{8,11}$ and endothelial activities. ${ }^{12-15}$

Recently, various optical diagnostic modalities, such as optical coherence tomography (OCT) ${ }^{16}$ Doppler OCT, ${ }^{17}$ correlation OCT ${ }^{18}$ laser speckle imaging, ${ }^{19}$ Laser Doppler flowmetry (LDF), ${ }^{20}$ have been developed and extensively used for functional studies of the skin microvascular system. Arguably, LDF is one of the most simple and popular approaches used in noninvasive diagnostics of blood flow in vivo. ${ }^{13} \mathrm{LDF}$ is based on the extraction of a phase shift (Doppler shift) from the detected signal caused by the scattering of probing laser light on the moving red blood cells proportionally to their velocity. Despite strong scattering of light by biological tissues, the Doppler shift can be measured with a reasonably high resolution. ${ }^{20}$

The current study applies the LDF approach ${ }^{20}$ to the study of skin blood microflow oscillations to assess the nature of changes in vascular tone in 32 (16 male and 16 female) healthy volunteers (HV) (average age $22 \pm 2$ year) and 60 (12 male and 48 female) PRD (average age $55 \pm 14$ year) from the Rheumatology Department of the Orel Regional Clinical Hospital, Russia. The study was approved by the local Committee for Human Biomedical Research Ethics, and all subjects signed an informed consent.

The measurements of blood microcirculation were performed using the experimental systems "LAKK-OP" and "LAKK-M" (SPE "LAZMA" Ltd., Russia). All procedures and measurements were approved beforehand by the Ethical Committee of the Regional Clinical Hospital. The LDF probe was placed on a skin pad (palmar surface) of the right middle finger, as shown in Fig. 1.

The LDF signal was collected during 5 min at normal conditions. During the cold pressor test (CPT), which was carried out for $5 \mathrm{~min}$, patient's arms were submerged in cold water with the temperature $\left(14 \pm 0.8^{\circ} \mathrm{C}\right)$ controlled by a contactless digital thermometer (Sensitec NB401, the Netherlands). The LDF signal was measured again immediately after retrieving arms from the cold water and after a 20-min relaxation. The results of a typical set of three LDF measurements are shown in Fig. 2.

The results of LDF measurements, shown in Fig. 3, show that at normal conditions PRD has a slightly higher perfusion rate $21 \pm 3$ p.u. compared to the HV: $18 \pm 4$ p.u. Significant variations occurred during the CPT: up to 7 p.u. for HV and 2 p.u. in the group of PRD.

The Mann-Whitney test was applied to quantitatively assess the difference. The obtained LDF signals were decomposed using a wavelet transform ${ }^{21}$ 


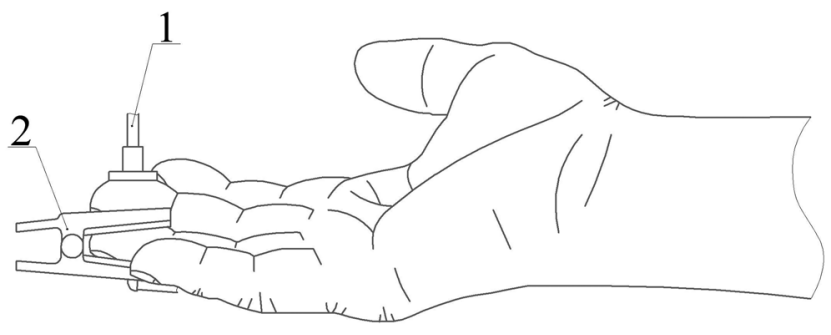

Fig. 1 Schematic presentation of the LDF probe positioning on a finger: (1) LDF probe and (2) pulseoximetry sensor.

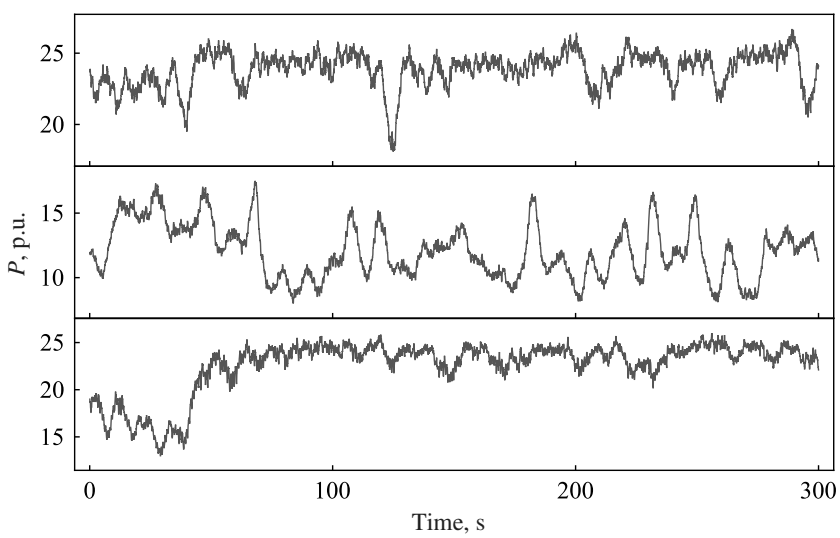

Fig. 2 (a) Example of measured LDF signals obtained at normal conditions, (b) immediately after cold exposure, and (c) 20 min after finishing CPT.

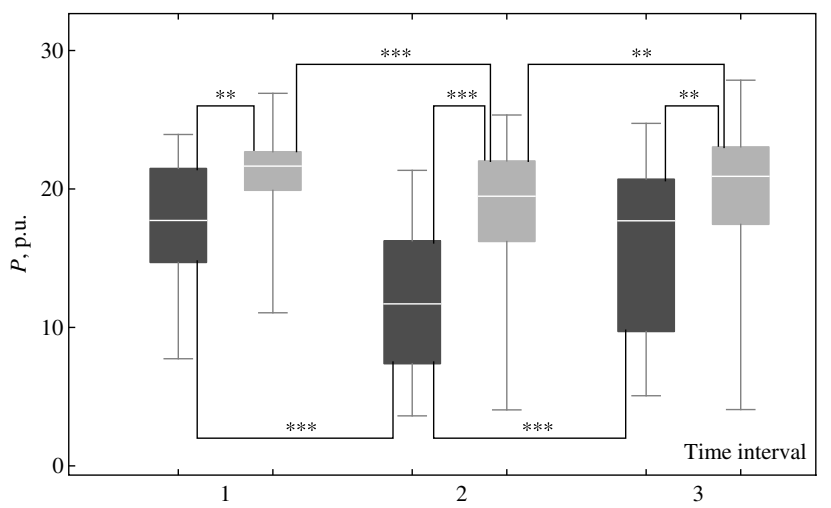

Fig. 3 Box-Whisker diagram of averaged perfusion: black rectangles correspond to the group of HV and gray ones to the group of PRD. Numbers indicate time intervals: (1) before CPT, (2) immediately after, and (3) 20 min after CPT. Stars indicate significance in the difference: $p<0.05, p<0.01$, and $p<0.005$.

$W_{x}(\nu, \tau)=\nu \int_{-\infty}^{\infty} f(t) \psi^{*}[v(t-\tau)] \mathrm{d} t$,

where * means complex conjugation. The Morlet wavelet, written in the form

$\psi(t)=e^{2 \pi i t} e^{-t^{2} / \sigma}$,

was used with the decay parameter $\sigma=1$. Integrating the power over time gives the global wavelet spectrum
$M(\nu)=\frac{1}{T} \nu \int_{0}^{T}|W(\nu, t)|^{2} \mathrm{~d} t$.

Being smoothed versions of Fourier transform, wavelets are known to be an effective tool for power spectral density estimation, especially for short nonstationary noisy data. Biomedical series are mainly short and nonstationary, and wavelet analysis is widely applied to trace energy distributions over frequencies in time. . $^{70,12,15,21}$

The wavelet coefficients were counted for the frequency band 0.01 to $2 \mathrm{~Hz}$ with the logarithmic partitioning on 50 frequencies. $M(\nu)$ was calculated for three records of LDFs (see Fig. 2) for each patient. The integral wavelet spectra were averaged over the group of patients. The energy distribution for
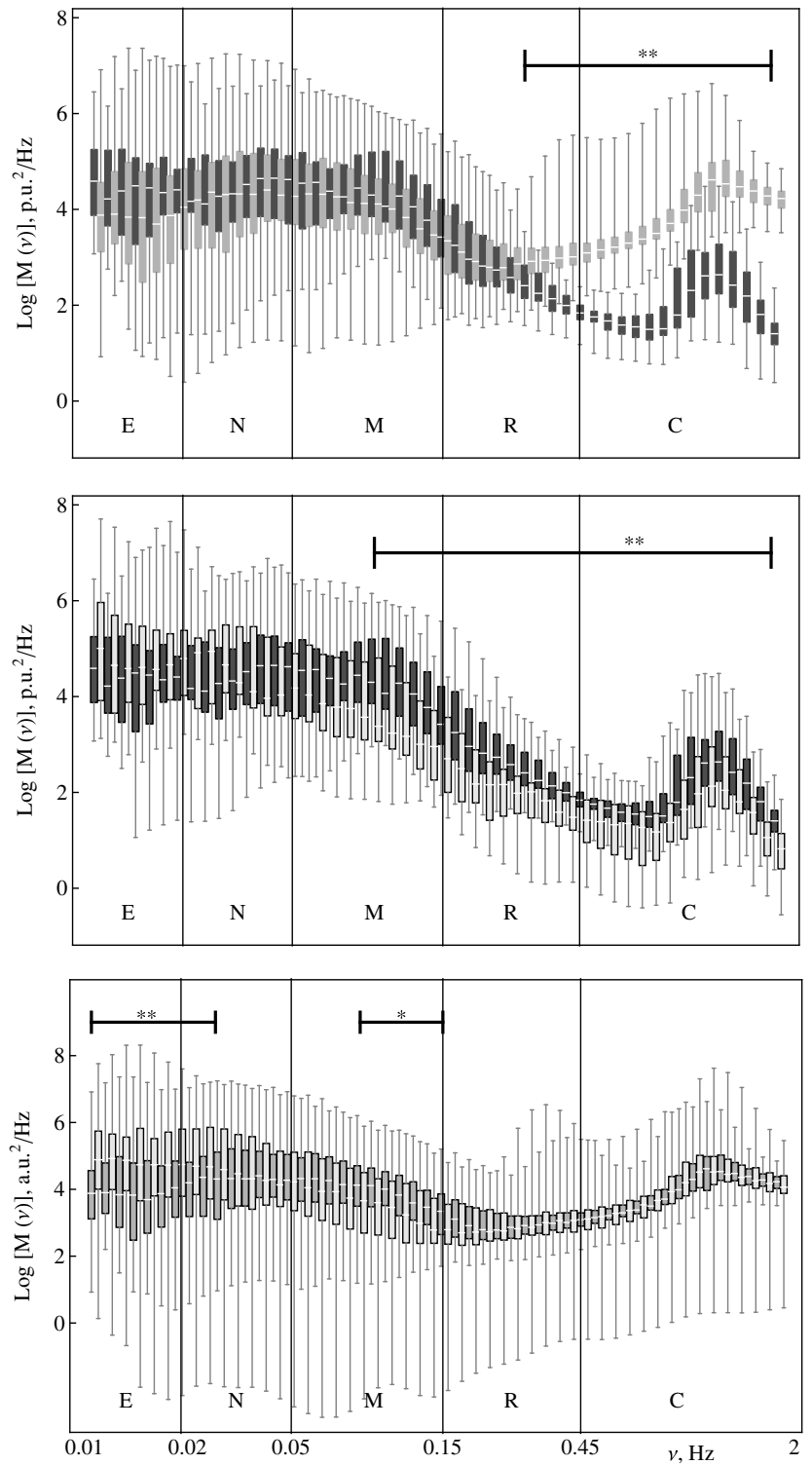

Fig. 4 Box-Whisker diagrams for the power spectral density: (top) for $1 \mathrm{HV}$ (black) and PRD (gray) at normal conditions; (middle) for HV only, taken at normal conditions (black) and after CPT (white); (bottom) for PRD only, at normal conditions (gray) and after CPT (white) at normal conditions. Solid stretches in the upper part of the plot indicate frequency bands where the difference between HV and PRD with pathology is significant. ${ }^{*}$ indicates $p<0.05$ and ${ }^{* \star}$ for $p<0.01$. 
every frequency band has been calculated. The results of a comparison of the distributions obtained for HV and PRD are shown in Fig. 4. The data processing was performed by Mathmatica 8.0, Wolfram research.

Figure 4 shows the wavelet spectra averaged over groups of $\mathrm{HV}$ and PRD at normal conditions. Higher frequency pulsations of the blood flow have been observed in the group of PRD compared to HV. The difference is significant up to $0.24 \mathrm{~Hz}$ $(p<0.01)$. At the same time, a trend to decrease an input of the low frequency oscillations is clearly indicated (see Fig. 4).

The analysis of averaged perfusion for HV and PRD showed significant differences before and after CPT (intervals 1 and 2) (see Fig. 3). The perfusion variation caused by cooling was more evident in the HV group. After 20 min of relaxation, the perfusion restored to the initial level. No significant difference was seen in the restore process (see Fig. 3, time interval 3), which agreed well with the results of alternative studies. ${ }^{22}$

We assume that higher perfusion and weaker response to CPT in the PRD group is associated with the abnormal behavior of the peripheral vascular bed, which is manifest in aptitude to the angiospasm. The significantly different spectral properties can be caused by morphological and functional abnormalities of the microvascular structures. We assume that the presence in microvascular structures of mega-capillaries ${ }^{23}$ and increased vessels stiffness decrease dumping properties of the circulation system with progression rheumatic pathology.

Finally, LDF has been successfully applied for the assessment of variations in blood microflows for PRD and HV. The obtained LDF signals have been analyzed utilizing a wavelet transform. The introduced approach provides an opportunity to obtain whole spectrum recordings of rhythmic processes in the microvasculature from pulse to circadian rhythms. Spectral properties of cutaneous blood flow vary due to the pathological process in the microcirculation system. The developed technique has a high potential in clinical application for functional evaluation of blood flow regulation and diagnosis of vascular abnormalities associated with rheumatic diseases.

\section{Disclosures}

No potential conflicts of interest relevant to this article were reported.

\section{Acknowledgments}

The work was supported by the Russian Foundation for basic research RFBR (projects no. 17-41-590560). Professor Meglinski also acknowledges partial support provided by the Russian Science Foundation (project no. 15-14-10008).

\section{References}

1. H. Gunawardena et al., "Maximum blood flow and microvascular regulatory responses in systemic sclerosis," Rheumatology 46(7), 1079_ 1082 (2007).

2. M. Gutierrez et al., "Capillaroscopic scleroderma-like pattern in patients without connective tissue disorders," Rheumatology 49(10), 1994-1996 (2010).
3. D. O'Reilly et al., "Measurement of cold challenge responses in primary Raynaud's phenomenon and Raynaud's phenomenon associated with systemic sclerosis," Ann. Rheum. Dis. 51(11), 1193-1196 (1992).

4. M. Rossi et al., "Skin vasomotion investigation: a useful tool for clinical evaluation of microvascular endothelial function?" Biomed. Pharmacother. 62(8), 541-545 (2008).

5. M. E. Mck-Weymann et al., "Respiratory-dependent laser-Doppler flux motion in different skin areas and its meaning to autonomic nervous control of the vessels of the skin," Microvasc. Res. 52(1), 69-78 (1996).

6. A. I. Krupatkin, "Cardiac and respiratory oscillations of the blood flow in microvessels of the human skin," Hum. Physiol. 34(3), 323-329 (2008).

7. A. Stefanovska, M. Bracic, and H. D. Kvernmo, "Wavelet analysis of oscillations in the peripheral blood circulation measured by laser Doppler technique," IEEE Trans. Biomed. Eng. 46(10), 1230-1239 (1999).

8. H. Schmid-Schönbein et al., "Synergetic interpretation of patterned vasomotor activity in microvascular perfusion: discrete effects of myogenic and neurogenic vasoconstriction as well as arterial and venous pressure fluctuations," Int. J. Microcirc. 17(6), 346-359 (1997).

9. A. I. Krupatkin, "Blood flow oscillations at a frequency of about $0.1 \mathrm{~Hz}$ in skin microvessels do not reflect the sympathetic regulation of their tone," Hum. Physiol. 35(2), 183-191 (2009).

10. A. I. Krupatkin, "The influence of the sympathetic innervation on the skin microvascular tone and blood flow oscillations," Hum. Physiol. 32(5), 584-592 (2006).

11. T. Söderström et al., "Involvement of sympathetic nerve activity in skin blood flow oscillations in humans," Am. J. Physiol. Heart Circ. Physiol. 284(5), H1638-H1646 (2003).

12. H. D. Kvernmo et al., "Oscillations in the human cutaneous blood perfusion signal modified by endothelium-dependent an endotheliumindependent vasodilators," Microvasc. Res. 57(3), 298-309 (1999).

13. M. J. Leahy et al., "Principles and practice of the laser-Doppler perfusion technique," Technol. Health Care 7(2-3), 143-162 (1999).

14. P. Kvandal et al., "Regulation of human cutaneous circulation evaluated by laser Doppler flowmetry, iontophoresis, and spectral analysis: importance of nitric oxide and prostaglandins," Microvasc. Res. 65(3), 160 171 (2003).

15. P. Kvandal et al., "Low-frequency oscillations of the laser Doppler perfusion signal in human skin," Microvasc. Res. 72(3), 120-127 (2006).

16. M. Bonesi, S. Proskurin, and I. Meglinski, "Imaging of subcutaneous blood vessels and flow velocity profiles by optical coherence tomography," Laser Phys. 20(4), 891-899 (2010).

17. M. Bonesi et al., "Application of Doppler optical coherence tomography in rheological studies: blood flow and vessels mechanical properties evaluation," J. Innovative Opt. Health Sci. 2(4), 431-440 (2009).

18. A. Doronin and I. Meglinski, "Imaging of subcutaneous microcirculation vascular network by double correlation optical coherence tomography," Laser Photonics Rev. 7(5), 797-800 (2013).

19. V. Kalchenko et al., "Label free in vivo laser speckle imaging of blood and lymph vessels," J. Biomed. Opt. 17(5), 050502 (2012).

20. E. A. Zherebtsov et al., "Combined use of laser Doppler flowmetry and skin thermometry for functional diagnostics of intradermal finger vessels," J. Biomed. Opt. 22(4), 040502 (2017).

21. P. Frick, I. Mizeva, and S. Podtaev, "Skin temperature variations as a tracer of microvessel tone," Biomed. Signal Process. Control 21, 1-7 (2015).

22. A. Waszczykowska et al., "Assessment of skin microcirculation by laser Doppler flowmetry in systemic sclerosis patients," Postep. Derm. Alergol. 1(1), 6-11 (2014).

23. M. Gutierrez et al., "Capillaroscopic scleroderma-like pattern in patients without connective tissue disorders," Rheumatology (Oxford) 49(10), 1994-1996 (2010). 